\title{
Understanding the Role of Mobile Ad hoc Networks in Non-traditional Contexts
}

\author{
Roberto G. Aldunate ${ }^{1,2,}$ Gregg E. Larson ${ }^{2}$, Miguel Nussbaum ${ }^{3}$, Sergio F. \\ Ochoa $^{4}$, Oriel A. Herrera ${ }^{5}$ \\ 1 Department of Civil and Environmental Engineering, University of \\ Illinois at Urbana Champaign, Urbana, IL, USA \\ aldunate@uiuc.edu \\ 2 Applied Research Associates, Inc., Champaign, IL, USA \\ glarson@ara.com \\ 3 Department of Computer Science, Catholic University of Chile, Chile \\ mn@ing.puc.cl \\ 4 Department of Computer Science, University of Chile, Chile \\ sochoa@dcc.uchile.cl \\ 5 Informatics Engineering School, Universidad Católica de Temuco, Chile \\ oherrera@uct.cl
}

\begin{abstract}
With the rapid development of short-range wireless technology new venues to apply it in more sophisticated, complex, and dynamic environments have been opened. Nevertheless, the applicability of such technology in nontraditional settings like face-to-face encounters and disaster relief environments, remains unclear. This article describes a research effort aimed to narrow that gap by means of using two non-traditional settings as case studies; face-to-face encounters among unacquainted people and first responders in urban disaster relief environments. Among the results obtained are: a) interactions among unacquainted people may be promoted, though the level of interaction becomes easily constrained due to the current state of RF technology and the design of the experiments, and b) it is feasible to obtain a reliable communication platform for first responders operating in disaster relief missions. These results supports the idea that short-range wireless technology may play both a facilitator and a promoter role in face-to-face contexts, and at least a facilitator role in the case of users co-located in highly dynamic contexts.
\end{abstract}

\section{Introduction: the role of MANETs in non-traditional settings is yet unclear}

With the emergence of short-range wireless technology, researchers and engineers have been enabled with a tool to explore, investigate, evaluate, and understand the applicability of Mobile Ad hoc Networks (MANET) in non-traditional complex and

Please use the following format when citing this chapter:

Aldunate, R.G., Larson, G.E., Nussbaum, M., Ochoa, S.F., Herrera, O.A., 2006, in IFIP International Federation for Information Processing, Volume 211, ed. Pujolle, G., Mobile and Wireless Communication Networks, (Boston: Springer), pp. 199-215. 
dynamic settings, like face-to-face spontaneous interaction or disaster relief environments, which have or may have high social and economic impact. MANETs have been envisioned by many authors as an attractive and feasible interaction means for mobile users in non-traditional contexts like the ones mentioned above.

The potential advantages of mobile ad hoc networking for areas without a suitable established infrastructure are well documented in research literature. For example, military environments are ideal for ad hoc networking and it has been recently noted that "today every advanced military is rolling out large-scale ad hoc networks to carry a variety of tactical communications traffic" [1]. Recently, much research is being conducted worldwide on using ad hoc networks in a variety of commercial applications, including emergency situations like fire-fighting and search and rescue operations where the probability of rescuing people trapped under rubble decreases as time passes after a disaster hit [2], inter-vehicle communications on roads, amusement parks, shopping centers, medical sensor monitoring, management at construction sites [3, 4], and many other sensor applications. Nevertheless, investigations on the MANET field have been mainly advocated to solve lower-level network problems, such as routing [5-11], following a technology-driven approach.

In spite of the high interest of the research community on MANETs, especially on lower-level network issues like routing and service discovery, the role that a MANET may play as facilitating and/or promoting interactions among mobile users in non-traditional settings remains unclear. The relevance of researching on non traditional and dynamic settings involving mobile co-located people is two-fold; at the same time that an increasing interaction or collaboration in these settings would facilitate the attainment of the goals of the people involved in such interactions, it would also help in understanding the boundaries for the applicability of infrastructure-less networks.

The rest of this article is structured as follows. Next section shows the research approach underlying this work. Section WiFi for Face-to-Face Encounters describes the investigation conducted to explore the facilitator and promoter role that this technology may play in such context. Section WiFi for Chaotic and Dynamic Contexts describes the work done on achieving a robust communication means for mobile first responders during disaster relief operations. The last two sections present a discussion regarding the research carried out and the conclusions and further work, respectively.

\section{Research Approach: MANETs for two non-traditional settings}

Current communication platforms or media used to support interaction between colocated mobile people in some non-traditional environments mismatch either satisfying highly demanding communication requirements or promoting the interaction among those people. Technologies such as sharing voice signal channels or semi-infrastructure solutions are adequate only for a reduced set of settings. In informal face-to-face interactions among unacquainted people the dependency on an infrastructure or a semi-infrastructure communication network is not natural because the interaction does not rely on information external to the actors, but on the information associated to and made available by each person. Face-to-face 
encounters among mobile users are considered in this research as an infrastructureless peer-to-peer network where such network is created dynamically as people moves. Therefore, potential face-to-face encounters will continuously require searching and matching profiles (stored in the wireless enabled devices carried by the people) of people nearby other people. Forcing this model to fit into a semiinfrastructure communication network could generate an extra overhead on such network; managing a roaming service to provide information about the geographic neighbors of each user. On the other hand, in chaotic, complex and inhospitable environments where fixed infrastructure communication is unreliable, such as in battlefield or disaster relief operations, shared voice signal channels and semiinfrastructure solutions constraint the potential collaboration among people involved in such task-oriented environments. Although several initiatives are pursuing development of wearable computers adequate for non-traditional settings, usually those researches are centered on the person (usability, storage, communication capability), but not on the characteristics that the communication medium should exhibit in such contexts.

This research effort is aimed at understanding the applicability of mobile ad hoc networks as a useful communication platform for co-located mobile users in non traditional and dynamic settings with high socio-economical impact. The approach used to carry out this research was based on selecting two settings as test-beds. Such settings represent very exigent contexts where the role of the communication medium requires to be evaluated. The specific problems addressed in this research are: (a) how to facilitate face-to-face interaction among unacquainted people in social settings, and (b) how to provide a reliable communication infrastructure for first responders in disaster relief environments. The general significance of this research is on gaining knowledge on principles, characteristics, design goals, mechanisms and algorithms that make a short-range wireless enabled communication medium adequate enough to improve interactions of co-located mobile users.

The core of the research was undertaken through a formal cycle of knowledge acquisition, formalization, systematization, and testing/validation and refinement. Nevertheless, different strategies for testing/validation were used for each one of the settings. For the case of face-to-face encounters among unacquainted people in everyday situations the solution developed was tested through experimentation. For the case of mobile co-located people involved in task-oriented, chaotic, inhospitable, and highly dynamic environments, simulations and experimentation were combined, inasmuch as simulations provided insight to this research about the applicability of mechanisms, algorithms and concepts previous to test them with real experiments, when it was possible given the limitation in reproducing the setting studied.

\section{WiFi for Face-to-Face Encounters: Facilitating \& Promoting}

The aim of this section is to describe how short-range wireless technology may facilitate face-to-face encounter among unacquainted people who are physically close and are (usually) moving in a given setting. In this way, people who do not know about each other's needs or knowledge could meet in a face-to-face scenario to establish a collaborative relation. The idea of Socialware is enhanced to a face-to- 
face Socialware. The communication/facilitator medium comprised 40 wireless interconnected Pocket PCs (IEEE $802.11 \mathrm{~b} / \mathrm{g}$ ) under distributed agent architecture. The developed interaction model was evaluated with freshmen engineering students from the College of Engineering of the Catholic University of Chile in their social and academic life. The main idea here was to provide mechanisms for the users to break the social contact threshold and ignite a relationship among people. Hence, the WiFi enabled communication medium was not only providing a communication bridge, but also could promote interaction between unacquainted students. When new students arrive to campus, it takes a while for the students to build trust among themselves, to know each other, form groups, work and study together, etc. To enhance student relationships, it is desired to foster face to face contact with people that are close to each other. Pocket PCs using WiFi permit formation of an Ad Hoc network between students that are within the network range (within 50 meters). Each student participating of the experience had a mobile device, where a software agent on the machine supports the user on email, chat, and peer search functions. The details of this work can be found in [12].

A study involving 180 first year students of Engineering and Psychology to measure their preferences and the impact of short-range wireless technology as facilitator of interpersonal attraction was conducted. Two questionnaires of more than 100 questions each were given to each student at the beginning and at the end of their first semester. A factorial analysis was performed to identify the latent variables or subjacent constructs among the observed inter-correlations from the different measured variables. The results showed five factors of preference: 1 - Shopping and/or leisure activities, 2 - Sport activities, 3 - Intellectual related activities, 4 Social activities, and 5 - Information activities. To identify patterns in the different profiles, a hierarchical accumulative cluster analysis was performed. This technique, based on the quadratic Euclidean difference as a measure of similitude, allows clusters to be identified that simultaneously present a high intra-group degree of similitude and a high inter-group degree of differentiation. Five clearly statistically significant $(\mathrm{p}<0,001)$ differentiable clusters were found.

\subsection{Results}

Figure 1.a illustrates the total transactions performed by the students Pocket PCs, registered by the eight PCs that monitored the networked during the semester. (It is interesting to mention that week six was an exam week). To understand this data, at the end of the experience and exhaustive questionnaire was applied to the participating students. Each of the question had to be graded from 1 to 7 ( 7 the best), being this the standard Chilean grading system that they were used to. Students claimed that the reasons for the below average scores shown on Figure 1.b were regarding technological issues, as shown in Figure 2.

Battery life was a key issue for the poor system usage. In this experience Toshiba e740 Pocket Pc were used, which have an integrated WiFi. Batteries lasted up to two hours when kept on. When people are on campus more than 6 hours daily, having availability of the machine is important. Battery life explains the level of usage of the tools for supporting encounters; students' machine 
intersection time is rather short (less than two hours) decreasing the probability of encounters. Students also claimed that the network coverage was also a problem. There were only eight wireless Internet gateways available on campus, each with IEEE $802.11 \mathrm{~b} / \mathrm{g}$ limited distance coverage. This distance coverage restriction was also a problem for the peer to peer connection. It showed that outdoors, as on a university campus, an operating range of up to 50 meters should be expected.

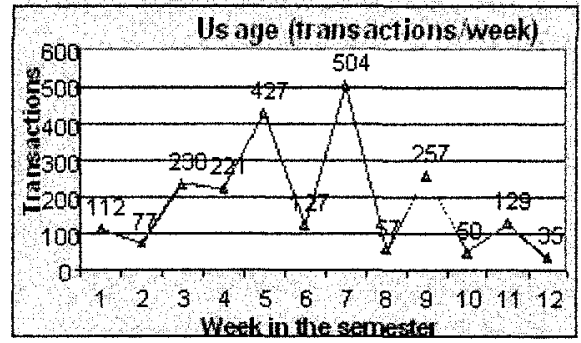

(a)

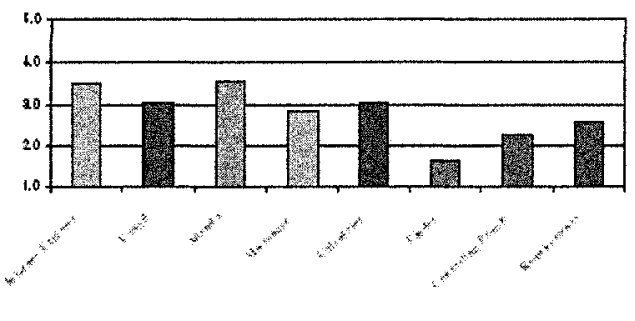

(b)

Fig. 1. (a) Students machine usage, (b) students functionality usage evaluation (1-7 scale)

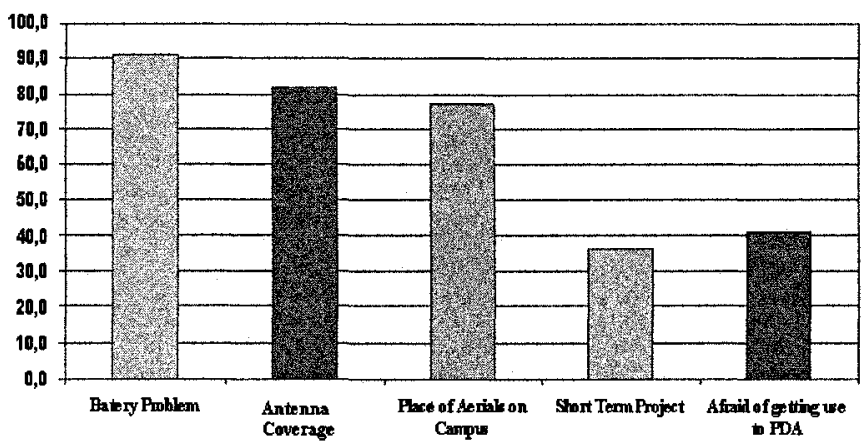

Fig. 2. Students problems usage evaluation

The first lesson learned through experimentation is that the high functionality provided to students played against their usability of the prototype. In addition, better battery technology is needed to improve the performance of the tools and improvements to the results of the study; the relatively scarce duration of the batteries in the experiments was the main obstacle in terms of communication. The results obtained in this work highlights that interactions were produced between people who were previously not familiar with each other, though the level of interactions was below the initial expectations of the undertaken research.

\section{WiFi for Chaotic and Dynamic Contexts: Facilitating}

To explore on the applicability of MANETs for chaotic and highly dynamic contexts, 
a disaster relief settings was chosen. For highly dynamic contexts like this, reliability is one of the key aspects to be addressed. To achieve a reliable MANET-based communication medium, the core of this work focused on achieving high availability and high connectivity in the MANET. Here, availability is understood as the capacity of the system to make its data, i.e., the data gathered and processes by the users of the MANET, fault tolerant despite the occurrence of varied types of failures. On the other hand, connectivity is understood as the capacity of the system to provide communication links among its members despite the occurrence of varied types of failures. (Detailed information about this issue can be found in $[13,14]$ )

\subsection{Availability: avoiding losing data}

A characteristic of a disaster relief operations scenario is that the first response teams communicate and collaborate among themselves using radio systems, because the fixed communication infrastructure usually is collapsed, unreliable or overloaded. Nevertheless, the voice channel based collaboration medium is limited in providing adequate support to collaborative efforts. Based on a literature review and comments obtained through interviews with expert civil engineers as well as firefighters participating in disaster relief environments, it is highlighted that radio systems tend to collapse in the early phases of first response process, because many people share few channels (usually 2 or 3 ) to interact with their partners [15]. MANETs have been envisioned by many researches as a potential solution for this problem. Nevertheless, for a MANET to be useful in such contexts, several issues must be addressed, availability being one of the primary ones, given the mobility of the users and the potential failures of links and devices in such a network.

\subsubsection{Redundancy}

For a MANET-based communication medium to provide high availability in contexts like disaster relief operations where failures are difficult or impossible to be avoided, data replication must be introduced. The amount of replication, i.e., how many times data is replicated, as well as the manner in which data is handled, i.e., the size of data chunks and its replicas, will affect the availability of the system. The approach used in this research to analyze data replication is based on the illustration given in Figure 3. The approach used in this part of the work is quite simple. The whole data space available at a MANET is portioned in data chunks which are replicated among the nodes comprising the MANET. Figure 4 shows that if data chunks are reduced, the probability a system failure, i.e.; a piece of data is lost from the system, increases. Specifically, in Figure 4.a, if Machine A fails at time t1 and Machine $C$ fails at time $\mathrm{t}$, the other members of the system, i.e.; Machines $\mathrm{B}$ and $\mathrm{D}$, will still have the whole data set. On the other hand, Figure 3.b, if Machines A and C fail, $\mathrm{A} 2$ and $\mathrm{C} 1$ data chunks will be lost from the system generating a system failure. The objective of the research on this topic is to determine how to structure the shared space to that the impact of node or link failures are reduced as much as possible on data availability. To understand the impact of such parameters, a simulation model based on the strategy described above was developed (a detailed description can be 
found in Aldunate et al., 2006a).

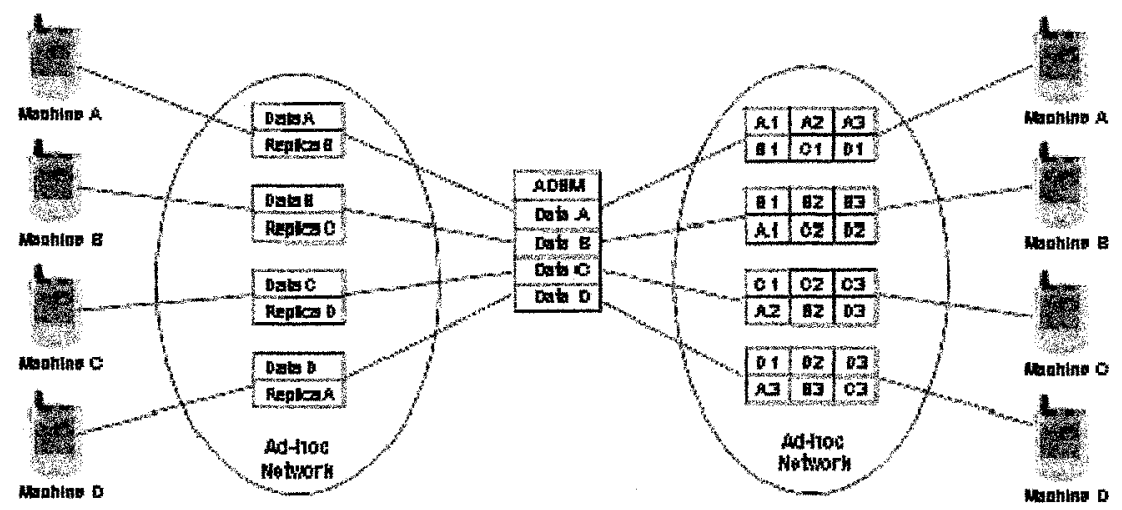

Fig. 3. Data replication example: (a) larger data chunks; (b) smaller data chunks

\subsubsection{Results}

During the first simulations (Figure 4.a), it was considered that 20 first responders including civil engineers would form a team (following FEMA, 1999 guidelines on the size of an Urban Search and Rescue Team [16]). In addition, the distance between two team members working in the disaster area (communication range) was initially set to be $50 \mathrm{~m}$, given our preliminary experimentation with IEEE $802.11 \mathrm{~b} / \mathrm{g}$ in semi-open environments, i.e., considering both inside buildings and outdoors. In order to assess availability for various disaster area scenarios and to identify the limits of the system, the size of the area was to change from $50 \times 50 \mathrm{~m}^{2}$ to $160 \times 160 \mathrm{~m}^{2}$. In addition, two sizes for the replicas were considered: Large Replica Units (LRU) where the size of each RU is defined as large as possible and Page Replica Unit (PRU) where the size of each RU is defined as a page, i.e.; 1-4 Kb. The simulation results demonstrated that the system's availability depends on the size of the replica; particularly, it decreases when the size of the replica unit decreases.

Moreover, high availability is obtained up to an area of about $130 \times 130 \mathrm{~m}^{2}$, if two or more large replicas are used. These results indicate that a team working alone using 2-LRU ( 2 Large Replica Unit) will be able to cover areas of $130 \times 130 \mathrm{~m}^{2}$, maintaining the availability of the system in the range of $98 \%$, when all the team members are inside the coverage area. Beyond that, extra support for communication will be required to avoid the isolation of some team members.

On the other hand, if the communication range is changed to $100 \mathrm{~m}$, high availability of $98 \%$ is obtained up to an area of about $250 \times 250 \mathrm{~m}^{2}$. It is clear that the larger the communication range, the larger the area will be that a first response team could cover while maintaining high availability of the system.

Additional simulations have shown that the availability of the system is also highly sensitive to the replication level introduced in the system and the number of team members. When a different number of replicas was used and each replica unit is defined as large as possible; Large Replica Units (LRU), it was noticed that the greater the replication level, the higher the availability. Finally, as expected, there is 
a positive correlation between availability and the replication level as well as the group size, as shown in Figure 4.b.

Once the platform was tested by computer simulations and the results demonstrated the feasibility of the system, the next step was to test it in a simulated disaster scenario. For that reason, a prototype was built to support a simulated search and rescue exercise. The evaluation of the system was carried out in parallel to the development of a normal search and rescue exercise conducted by the Illinois Fire Service Institute (IFSI) of the University of Illinois at Urbana-Champaign at its training facilities. The setting for this exercise included two office buildings and a pile of rubble, which simulate partial and total collapses, twenty-four apprentice firefighters and five people testing the system in situ collaborating with one remote structural expert. They assumed the roles of rescuers, team leader, and local and remote structural experts. The exercise was monitored by an expert in search and rescue operations.

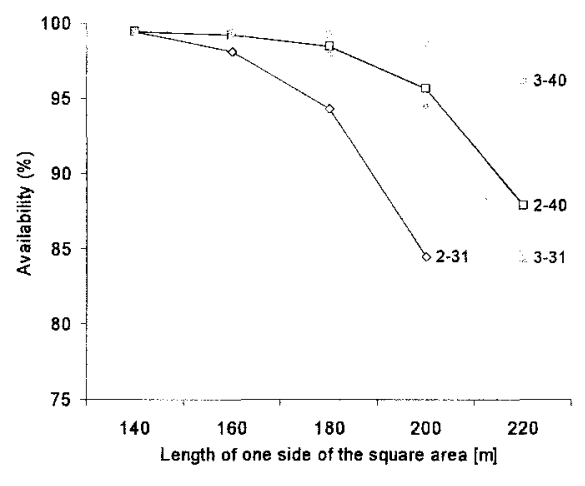

(a)

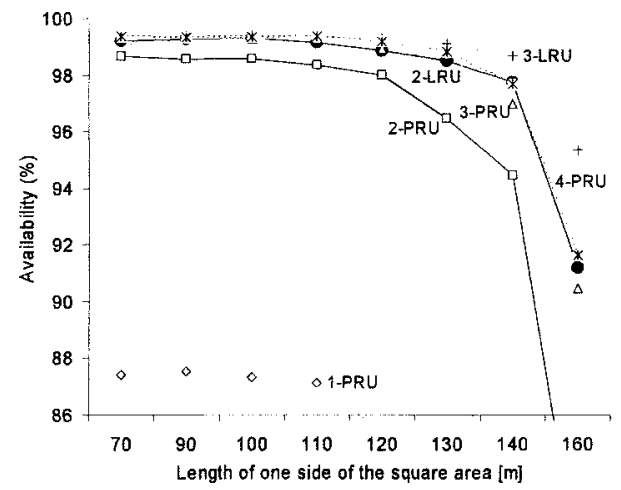

(b)

Figure 4-System's availability depending on the size of the data chuck and the size of the operations area size for: (a) 20 team members and a communication range of $50 \mathrm{~m}$; and (b) a communication range of $50 \mathrm{~m}$ and variable level of replication and number of members

The outdoors results outperformed the availability results of simulations runs, because of the difference between the empirical wireless communication range with the estimation made for simulations; $200 \mathrm{~m}$ v/s $50-100 \mathrm{~m}$. In addition, although indoor communication range was highly variable depending on number and composition of walls, the system remained always available during the testing. Overall, it was observed that the difference between outdoors and indoor communication range is large, but the replication strategy reduced effectively the impact of this parameter on the system's availability. Another difference between the simulations and the experiment, which also explains the better availability results obtained during experimentation in comparison to simulations, is related to first responders' movement behavior. In the real scenario, the first responders moved in small groups using predefined paths, not randomly as was considered in simulations, decreasing the probability of network disconnections. 


\subsection{Connectivity: avoiding losing users}

The next step towards determining the usefulness or applicability of MANETs for highly dynamic contexts is dealing with connectivity problems. The previous topic covered the issue of data availability in spite of users getting disconnected from the MANET. Nevertheless, in a real complex setting, like disaster relief operations or battlefield, it would be desirable to avoid as much as possible such disconnections. Maintaining connectivity within a MANET presents a challenge due to the fact that communication between short range wireless enabled communication devices can be interrupted by physical objects, environmental conditions affecting the radio frequency signal, hardware, software, and connection lost due to the mobility of users, among others. The connectivity problem due to the mobility of mobile users is considered by this research as the key factor for the development of the proactive nature of the mechanism. All the other causes of failure are considered as a whole in a parameter referred to as Link Failure Probability used in the analysis of the selfhealing mechanism in the following sections.

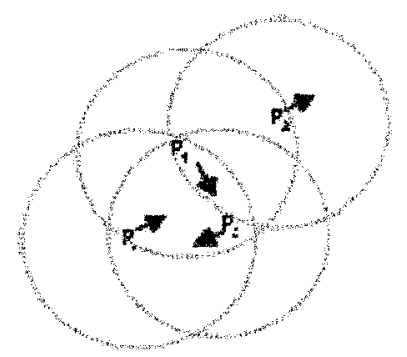

Figure 5 - MANET $=\left\{P_{1}, P_{2}, P_{3}, P_{4}\right\}$ will become fragmented, Set $1=\left\{P_{1}, P_{3}, P_{4}\right\}$ Set $2=\left\{P_{2}\right\}$

The general connectivity problem generated by the mobility of mobile users is represented through the situation illustrated in Figure 5 . In that figure $P_{1}, P_{2}, P_{3}$, and $\mathbf{P}_{4}$ represent mobile users who communicate with each other using short-range wireless enabled devices, at a given time. Arrows represent the direction of movement for each mobile user. Thus, considering the direction of movement of each mobile user shown in Figure $5, \mathrm{P}_{2}$ will eventually leave the communication range of $P_{1}$, and vice-versa. In addition, $P_{3}$ and $P_{4}$ will remain within the communication range of each other, and also within the communication range of $P_{1}$. In general terms, this situation represents network fragmentation; i.e., subsets of the original MANET can not communicate each other. Depending on the movement behavior of the nodes comprising the MANET, network fragmentation can be either permanent, e.g., some fragments of the network do not enter into communication during the period of operation, or temporary; i.e., the fragments merge again at a further time during the period of operation.

\subsubsection{Self-Healing Mechanism}

To cope with the problem described above, unmanned vehicles enabled with communication devices similar to those used by the mobile users are introduced. 
This idea is based on Darpa's Minefield Project [17], though the approaches are very different (see [14]). The unmanned vehicles, here referred to as Mobile Communication Bridges (MCB), should be able to detect "disconnection in progress" messages spread by $\mathrm{P}_{2}$ and $\mathrm{P}_{1}$ (Figure 5) and move accordingly toward the region between them, avoiding a disconnection or reducing the disconnection time as much as possible, in case it was not possible to reach the disconnection area on time. This approach is opposed to deploying static repeaters, which one can envision might be a straightforward solution for small areas, but it might not scale for very large areas.

An underlying problem to address under this topic is message delivery. The message delivery service in the designed system is based on ad hoc gossip multicast [11], because it offers a solution in between routing and flooding techniques. By using a probabilistic multicast technique, like gossip-based multicast, this research expected the healing mechanism achieves high reliability with moderate degradation of performance.

The self-healing mechanism relies on the following three-phase algorithm:

Potential Disconnection Detection: this phase is performed permanently by every node in the MANET; i.e., mobile users and MCBs. At every time step $t$, each node monitors its 1-hop neighbors. For each 1-hop neighbor that is located at a distance greater than a certain threshold, given as a parameter, the monitoring node records such distance at that moment. At next time step, the monitoring node will determine the new distance for each previously monitored 1-hop neighbor. If, for each 1-hop neighbor, the distance has increased, the monitoring node will try to find out if at least one of its other 1-hop neighbors is closer to the one under observation. If the monitoring node fails on such task, it will propagate a "potential disconnection" message through the MANET.

Correction: this phase is also implemented by both mobile users and MCBs. Once a node detected a potential disconnection, it will propagate a message through the network, using gossip-based multicast. Any MCB receiving that message will wait a given period of time for the counterpart message; i.e., the message sent by the other node involved in the potential disconnection. If the counterpart message is received by the $\mathrm{MCB}$, it will ignore the situation because it means that there is at least one alternative route connecting both nodes. But, if the counterpart message is not received by the $\mathrm{MCB}$, it assumes a disconnection is in progress and it will set itself toward that task; i.e., move toward the potential disconnection area.

Maintenance: this phase of the algorithm is applicable only to MCBs; i.e., not applicable to devices used by mobile users. Once the MCB is placed at the potential disconnection area, it tries to detect the presence of the requesting nodes. If the MCB does not find the requesting nodes after a given period of time, it will set itself back to Idle state. Nonetheless, if the MCB detects the requesting nodes, it sets its state to Supporting mode, adjusting its position dynamically based on the movement of the supported nodes. The MCB will remain in Supporting mode, until either the supported nodes are again in communication range or the supported nodes are apart and the disconnection is imminent. In such case, the MCB will play the potential disconnection phase. 
The three-phase algorithm and the principles presented rely on two assumptions. On one hand, the set of MCBs is comprised of homogenous devices not only in terms of communication capability, but also in terms of mobility; i.e., they are able to move at similar speeds. On the other hand, it is assumed the environment is free of obstacles; i.e., the time for a MCB to reach a target location depends only on the distance to such area.

The correctness of the above presented algorithm; i.e., at least and at most one MCB will support a disconnection when there are one or more MCBs in Idle state, can be guaranteed only if the following special conditions are met: (a) the message delivery service is $100 \%$ reliable, and (2) the underlying location service is $100 \%$ reliable. If the former service is not completely reliable, it could be possible no MCB in Idle state hears about a potential disconnection call or that two or more MCBs "accept" one mission unaware of their concurrent movements. If the latter service is not completely reliable, the distance estimations a MCB makes while in Moving state could be decreasing no monotonically, and so, it could not be guaranteed the closest MCB is moving to the disconnection area where the disconnection call was generated. As the current state of development for both services; i.e., technology, can not guarantee fully reliable services, the three phase algorithm is not able to guarantee an optimal solution, e.g., two or more MCBs heading to a disconnection area could become aware of the race condition only when they are close to the target location (at that point, the Correction Phase solves this conflict), but a best effort solution.

During discrete-event simulation runs first responders are modeled as moving pseudo-randomly, with a probability for them to stay stationary for some time intervals, and failures like technology dependant network failures, failures of electronic components due to fatigue of material, battery consumption, software bugs, and death or accidents of first responders are modeled through a Link Failure Probability (LFP) factor. Also, MCBs are in one of three modes: (1) Idle, (2) Moving towards a specific area or (3) Supporting Communication. The Idle mode represents a stationary MCB waiting for messages indicating a potential disconnection is in progress. The Moving mode is straightforward up to the moment when it reaches the area near the requiring pair of nodes, then, it will check if any other node is acting as a bridge for those nodes. If this is the situation, it will set itself to Idle mode, otherwise will set to Supporting Communication mode. The Supporting Communication mode represents a $\mathrm{MCB}$ which has reached the pair of nodes requiring a communication bridge. In that situation, a MCB is considered to be able to determine the area between the nodes requiring connectivity by using some proximity or triangulation technique, like GPS. While in the Supporting Communication mode, the MCB will evaluate new positions of the pair of nodes for which it is providing connectivity. With that information, the MCB will reevaluate its area of operation; i.e., nearby the point at the middle of the line representing the Euclidean distance between the nodes supported.

\subsubsection{Results}

The results obtained highlight the relationship between the number of mobile users (excluding MCBs) and connectivity, as shown in Figure 6. It can be appreciated that connectivity positively correlates with the number of mobile users. It can also be 
appreciated that for a team of 30 mobile users operating in area of $400 \times 400 \mathrm{~m}^{2}$, the number of disconnections are approximately 12; i.e., the MANET becomes highly fragmented. Nevertheless, the most important result shown in Figure 6 is that to achieve high connectivity in the MANET, al least 70 mobile users should operate in such area. A different way to understand the results shown in Figure 6 is that if high connectivity is required for a team of 30 mobile users in the defined area of operations, at least $40 \mathrm{MCBs}$ without coordination and pseudo-random movement pattern should be deployed. Such strategy implies that MCBs are always active, reducing the chances for energy saving. Several other aspects of the dynamics of the self-healing system were observed (the reader can find them in [14]), nonetheless the most relevant is the relationship between Link Failure Probability (LFP) and the reliability of the self healing mechanism, as shown in Figure 7. For LFP $<0.7$, the MCBs are mainly in Supporting mode, while for a LFP $>0.8$ the MCBs mainly are in Idle mode. When $0.7<$ LFP $<0.8$, the system suddenly experiences a phase transition. In other words, the self-healing mechanism becomes useless for values of LFP $>=0.75$. This dynamics is analyzed in the next section.

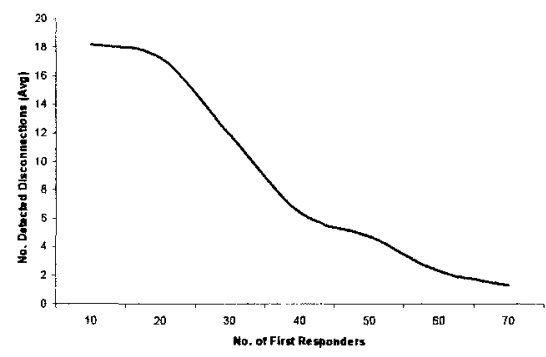

Figure 6. The number of disconnections decreases linearly as the number of first responders increase in an area of $400 \times 400 \mathrm{~m}^{2}$, without participation of $\mathrm{MCBs}$

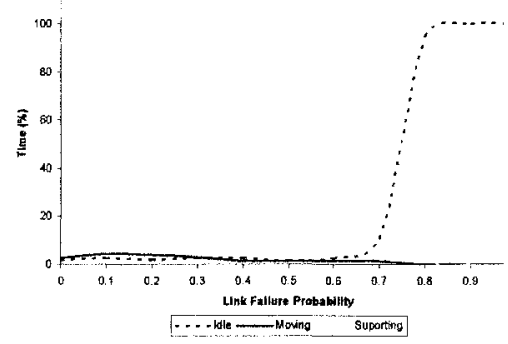

Figure 7. MCBs exhibit high power consumption and low percentage idle up to a link failure probability of about 0.7 . After that value, they become stationary

\subsubsection{Mathematical Analysis}

To better understand the dynamics shown in Figure 7, a mathematical analysis was conducted. Based on the diagram presented in Figure 8 , and considering as transition probabilities $p_{s x}=$ Probl( nodes supported by the MCB become able to communicate without the support of the $M C B$ ) $O R$ (MCB does not receive answer from supported nodes to request messages generated by the $M C B)]$; as $p_{\mathrm{sx}}=\gamma(1-L F P)^{2}+L F P^{2}$, and $p_{x s}=\operatorname{Prob}(M C B$ in Idle state receives potential disconnection messages \& $M C B$ is "chosen" to support potential disconnection informed); $p_{x s}=\lambda(1-L F P)$, the plot shown in Figure 9 is obtained.

Although the main purpose of the analytical model developed in this section is to obtain a qualitative view of the dynamics of the system, in contrast to the quantitative and detailed view obtained through simulations. First, not surprisingly, for a MANET comprised of mobiles users which has a permanent demand for MCBs to provide connectivity, the self-healing mechanism will tend to collapse as the reliability of the communication decreases; i.e., a link probability failure greater than 
0.7. Put in other way, the more reliable the message delivery used for the nodes of the MANET to communicate; i.e., mobile users as well as MCBs, the more efficient the utilization of MCBs. Also, the self-healing mechanism will require the MCBs spending more time in Supporting mode in settings where the probability of reencounter (communication range) between nodes communicated through MCBs; i.e., $\gamma$, is lower. Regarding vulnerability, for the self-healing mechanism to be less vulnerable to link failures, the proportion of MCBs in Idle (\& Moving) state should be kept as close as possible to the demand for supporting disconnections; i.e., $\lambda \rightarrow 1$. Finally, a very interesting outcome is that the dynamics of the self-healing mechanism obtained through the analytical model which considers a large number of MCBs, and consequently a very large number of mobile users, is similar to the dynamics obtained from simulation runs considering small scale MANETs.

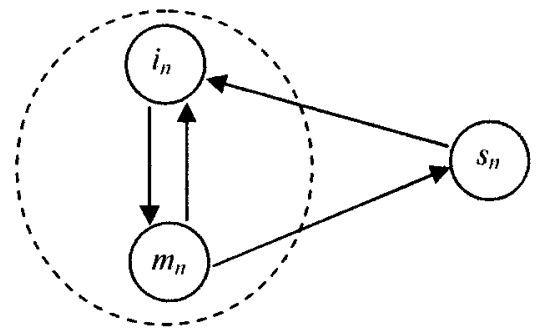

Figure 8. States diagram representing the proportion of MCBs in each state; i.e., $i=$ Idle; $m=$ Moving; and $s=$ Supporting. States $i$ and $m$ comprise the state $x$, and $p_{i j}$ represents the transition probability, per round, from state $i$ to state $j$.

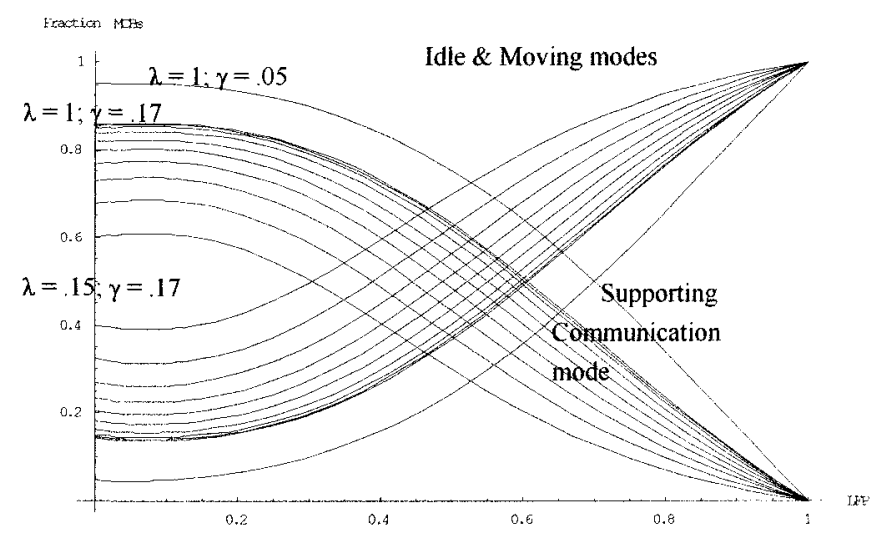

Figure 9. Impact of Link Failure Probability (LFP) on the attractors of the dynamics of the self-healing mechanism; a different pair of curves is obtained for different values of $\lambda$ (the rate between the number of disconnections perceived by MCBs and the number of MCBs available to respond to disconnection calls). The lower the value of $\lambda$, the lower the LFP threshold on which the phase transition would occur. The LFP threshold for a phase transition to occur corresponds to the $\mathrm{x}$ value at the intersection of the pair of curves (decreasing curve represents Supporting mode, increasing curve represents Idle \& Moving modes) for each $\lambda$. 


\section{Discussion}

In addition to the encouraging results obtained, several issues arose regarding the applicability of MANTEs for the non-traditional contexts studied. For example, one design decision to experiment among unacquainted freshmen considered incorrect was the high degree of functionality provided to them. In opposition to initial expectations, high functionally limited the use of the system to interact with other unknown students. Another problem discovered during the experimentation in promoting face-to-face encounters was connectivity to the fixed infrastructure. Although the fixed infrastructure portion of networking was only used only for data gathering and monitoring, and not for connectivity between freshmen, the students claimed for the intermittent Internet service provided through the MANET. As only intermittent connectivity was possible between the peer-to-peer network and the fixed infrastructure; problems of reliability in monitoring and data collection processes were experienced due to the communication range of IEEE $802.11 \mathrm{~b} / \mathrm{g}$. This problem relies basically on the decision of not introducing a routing mechanism, because multi-hop was against the idea of face-to-face interactions.

Another relevant issue related to the wireless technology used, IEEE802.1b/g, is the communication range, especially in indoor environments. During experiments the indoors communication range can dramatically be reduced depending on the nature of the objects interfering line of sight between the wireless enabled devices. These problems require that the design has to deal with the availability of the communication infrastructure, which could be addressed by adding replication or adequate communication mechanisms to maintain communication.

Also, battery duration was a key constraint for the experiments conducted; tasks where frequently interrumpted to replace batteries every couple of hours. Battery usage is determined by the energy consumption of the device and specifically the consumption of the wireless interface. Thus, a trade-off between battery duration and functionality arises. The more the functionality provided by the device and bandwidth provided by the wireless interface, the less battery duration, e.g., cellular phone batteries last longer than PDAs batteries, but with limited wireless bandwidth and consequently limited applications. It must be stressed that the short battery duration limits the current use of this technology in disaster relief environments. One possible solution for this constraint is the use of wearable computers which have strong battery duration and backup specially designed for the reliability of continuous operation of first responders and other users in infrastructure-less scenarios, such as a battlefield or on-site construction. However, this solution affects usability because it imposes more weight to be carried by the user.

Regarding disaster relief environments, one of the most disputable assumptions made could be the establishment of and scenario without obstacles. In real disaster relief environments the density, layout and structural status of the infrastructure will depend on the material as well as the construction processes utilized to build the physical infrastructure. Thus, a paramount effort would be required to be able to classify types of scenario from a detailed perspective. Nevertheless, this research effort did consider them; the impact of most of the factors which may generate 
communication failures was modeled as an general failure parameter introduced in both the availability and connectivity analises.

A second important assumption made related to the scenario of first responders is that they move pseudo-randomly in the field. Nevertheless, the movement of people in a real setting is not envisioned as random, but task oriented. Further research is required on this aspect to evaluate the impact of this factor.

Regarding the structure of the platform to supporting interaction among mobile users in chaotic and highly dynamic contexts, this research focused on achieving an autonomous communication platform. A open question is how to integrate this research with research efforts dealing with connectivity to infrastructure networks when they are available. MANET-based networks used in urban search and rescue contexts, could play the (analogous to a cognitive memory model) role of short term memory, inasmuch as long-term memory could rely on a fixed infrastructure communication platform. Specifically, one of the possible uses of this platform is to be engaged with a fixed infrastructure (or satellite) to provide communication between on-site users with remote users which would assist them in analyzing data, developing adequate problem analysis of ongoing situations, as well as making realtime solutions and decisions, among others.

In terms of usability one must face the question: to what extent would the devices really be used by the users? In the case of informal social encounters among unacquainted people, usability is not envisioned by us as a challenging problem in future due the growing popularity in the usage of handheld devices on the population, especially among young users. In opposition, in the case of first responders, usability is envisioned to be a challenging issue to solve, due to the imperious necessity of develop adequate interfaces to avoid that the medium becomes a cognitive or physical obstacle for the development of ongoing tasks. This article describes research focused on the technological feasibility of short-range wireless technology used to provide communication medium for first responders' collaboration in disaster relief environments, i.e.; focused on aspects are related to machine-machine communication and neither human-machine or human-human relationships.

Finally, the general approach taken by this research effort for the development of algorithms, methods and techniques was heuristic and distributed. Although in the academic environment it was possible to attach the MANET to an infrastructure network, which was mainly used to monitor interactions between people, but not to provide interaction between students, in the disaster relief environment case it was modeled completely in a distributed manner. Optimal analytical solutions would be more difficult, if not impractical at all, due to the mostly partial information handled by the users in chaotic and highly dynamic contexts [18]). Consequently, the solutions proposed for the availability and connectivity problems stated in this document are "best-effort" solutions. 


\section{Conclusions and Further Work}

The research effort presented through this document was aimed to understand to what extent short-range wireless mobile networks are a useful medium able to support interactions among co-located mobile people in extreme interaction settings which have or may have a high social impact. The semantics of extreme setting in this document is defined as an interaction context where the level of exigency for the agent mediator of interactions, i.e., the communication medium, is far beyond the levels of exigency of settings such as formal face-to-face work meetings. In order to do this, two settings were chosen; face-to-face interactions among unacquainted people in everyday situations, and first responders communication in urban disaster relief environments. In the former setting, the medium was studied in terms of the role as facilitator/promoter of such face-to-face interactions. The communication between physically close people, e.g., a couple of dozens of meters, even if it is intermittent, is not an issue due the current available off-the-shelf short-range wireless technology. On the contrary, in the latter settings, the communication is the primary issue to deal with. Such priority is given by the need to provide the first responders with an autonomous, functionally adequate, and reliable communication medium for inhospitable, complex, chaotic and dynamic environments where fixed infrastructure computational networks are unreliable, or physically or logically collapsed.

The main conclusion of this research effort is that short-range wireless mobile networks can play key roles for extreme interaction settings involving co-located mobile people. The results obtained in this research effort, through experiments and simulations, show that: (a) a short-range wireless mobile network may promote faceto-face encounters among co-located unacquainted people in everyday situations, and (b) a short-range wireless mobile network may be established as a reliable communication medium to support interactions among first responders operating in complex, chaotic, and highly dynamic contexts.

Through the development of this research effort some of the leading original questions were answered, some of them only could be partially answered, and new ones rose. To what extent it is possible to generate long-term relations among unacquainted people by promoting their face-to-face initial contact through technology? What kind of interactions is more prone to be promoted by a technological promoter? How many different environments could exist in disaster relief operations considering the different nature of natural and man-made disasters? What are the most useful first responders movement models for each kind of disaster relief setting? Is it possible that technology plays adequately the role of promoter for stressing, hostile, chaotic, and dynamic disaster relief environments? How applicable are the concepts, principles, models, mechanisms, and algorithms developed through this research to other slow or business contexts? These and other questions will lead further research. 


\section{References}

1. Elliot, C. (2003) "U.S. military pioneers ad hoc wireless networks in the battlefield." iApplianceWeb.com, http://www.iapplianceweb.com/story/OEG20030319S0054.

2. Yusuke, M. (2001) Collaborative Environments for Disaster Relief. Master's thesis, Department of Civil \& Environmental Engineering, MIT, Cambridge, MA, June.

3. Amagar, S. and Hayashi, M. (2002) "Ad Hoc Networks: Applications and Merits." ETSI Workshop on Broadband Wireless Ad-Hoc Networks and Services, Sophia Antipolis, France, September.

4. Kirisci, P.T. (2002) "Context-Aware Environments for Ad Hoc Collaborative Business," Technology Challenges Workshop, Stuttgart, Germany, October.

5. Johnson, D., and Maltz, D.A. (1996), "Dynamic Source Routing in Ad Hoc Wireless Networks." Mobile Computing, Vol.353, T. Imielinsky and H. Korth, eds., Kluwer Academic Publishers, January 1996, Boston, MA, chapter 5, pp. 153-181.

6. Dube, R., Rais, C.D., Wang, K., and Tripathi, S. (1997) "Signal Stability based Adaptive Routing (SSA) for Ad-Hoc Mobile Networks." IEEE Personal Communication, Feb., pp. 36-45.

7. Broch, J., Maltz, D.A., Jonhson, D.B., Hu, Y.C., and Jetcheva, J. (1998), “A Performance Comparison of Multi-hop Wireless Ad Hoc Network Routing Protocols." Proc. Fourth Annual ACM/IEEE International Conference on Mobile Computing and Networking, October 25-30, 1998, Dallas, TX, ACM, New York, NY, pp. 85-97.

8. Perkins, C.E., and Royer, E.M. (1999) "Ad Hoc on Demand Distance Vector Routing." Proceedings 2nd IEEE Workshop an Mobile Computing Systems and Applications, February 25-26, 1999, New Orleans, LA, IEEE, Washington, DC, pp. 90-100.

9. Royer, E.M. and Toh, C.K. (1999) "A Review for Current Routing Protocols for Ad Hoc Mobile Wireless Networks." IEEE Personal Communications, April, pp. 46-55.

10. Nasipuri, A., Castañeda, R. and Das, S.M. (2001) "Performance of multipath routing for on-demand protocols in mobile ad hoc networks," Mobile Networks and Applications, Vol 6, pp.339-349, August.

11. Haas, Z., Halpern, J., and Li, L. (2002) "Gossip-Based Ad Hoc Routing." In Proceedings of IEEE Infocom 2002.

12. Nussbaum, M., Aldunate, R., Sfeir, F., Oyarce, S., and Gonzalez, R. (2004) "Ubiquitous Awareness in an Academic Environment." Lecture Notes in Computer Science, SpringerVerlag Heidelberg, Volume 2954 / 2004, pp. 244-255.

13. Aldunate, R., Ochoa, S.F., Pena-Mora, F., and Nussbaum, M. (2006a) "Robust Mobile Ad Hoc Space for Collaboration to Support Disaster Relief Efforts Involving Critical Physical Infrastructure." Journal of Computing in Civil Engineering, Vol. 20, Issue 1, pp. 13-27.

14. Aldunate, R., Pena-Mora, F., Gupta, I., Gasser, L., Nussbaum, M., and Ochoa, S. (2006b) "Self-healing Communication for Mobile Ad-hoc Networks in Inhospitable Context." Under review in IEEE Transactions on Systems, Man and Cybernetics - Part C: Applications and Reviews.

15. Jackson, B., Peterson, D., Bartis, J., LaTourrente, T., Brahmakulam, I., Houser, A., and Sollinge, J. (2001). "Protecting Emergency Responders: Lessons Learned from Terrorist Attacks." RAND, Science and Technology Policy Institute

16. Fema (1999) "Federal Response Plan." Federal Emergency Management Agency, 9130.1PL. April

17. Altshuler T. (1999) "Opportunities in Land Mine Warfare Technologies. The Self-Healing Minefield." (Approved for Public Release, Distribution Limited) Defense Advanced Research Projects Agency (DARPA), Advanced Technology Office, Arlington, VA

18. Gasaway, R. Ready! Fire! Aim!. Journal of Fire Engineering, pp.16-17. August. 2003 